\title{
Intervención didáctica tecnológica para el estudio de las secciones cónicas basada en el potencial semiótico
}

\author{
Sonia Valbuena-Duarte ${ }^{1,{ }^{*}}$, Yilmar Tamara-Gutiérrez ${ }^{2}$ y Jesús D. Berrio-Valbuena ${ }^{3}$ \\ (1) Programa Lic. en Matemáticas, Universidad del Atlántico, Grupo GIMED, Barranquilla-Colombia. \\ (correo-e: soniabalbuena@mail.uniatlantico.edu.co) \\ (2) Nuestra Señora de la Candelaria. Atlántico-Colombia. (correo-e: tamarayilmar@gmail.com) \\ (3) Programa Lic. en Matemáticas, Universidad del Atlántico, Grupo GIHEM, Barranquilla-Colombia. \\ (correo-e: jberriovalbuena@mail.uniatlantico.edu.co)
}

* Autor a quien debe ser dirigida la correspondencia.

Recibido Jun. 23, 2020; Aceptado Ago. 24, 2020; Versión final Sep. 15, 2020, Publicado Feb. 2021

\begin{abstract}
Resumen
El objetivo del presente estudio es identificar, analizar y caracterizar los registros semióticos en el estudio de las secciones cónicas con profesores en formación. La metodología con enfoque mixto, diseño basado en ingeniería didáctica y corte cuasi experimental es desarrollada por fases: 1) análisis preliminar de concepciones y formas de enseñanza, 2) diseño a priori e implementación del recurso, y 3) análisis a posteriori. Los resultados evidencian dificultades de aprendizaje y enseñanza acentuadas en la actividad cognitiva de conversión. Por tanto, se diseña e implementa una intervención didáctica tecnológica basada en un recurso educativo digital de acceso abierto, apoyado en la teoría de los registros de representación semiótica de Duval. Se concluye que el recurso es mediador para el estudio de las cónicas y la adquisición de nuevos conocimientos, adicionalmente permite la movilización de saberes matemáticos desde perspectivas diferentes al enfoque gráfico o al lenguaje común.
\end{abstract}

Palabras clave: recurso educativo digital; registros semióticos; ingeniería didáctica; secciones cónicas

\section{Technological didactic intervention for the study of conical sections based on semiotic potential}

\begin{abstract}
The objective of the present study was to identify, analyze, and characterize semiotic registers of conic sections by teachers in training. The methodology used a mixed approach based on didactic engineering. The quasiexperiment was developed in three phases: 1) preliminary analysis of conceptions and forms of teaching, 2) a priori design and implementation of educational digital resources, and 3) a posteriori analysis. The results showed learning and teaching difficulties, especially in the cognitive conversion activity. A technological didactic intervention was designed and implemented based on an open access digital educational resource supported by Duval's semiotic representation theory of registers. It is concluded that the resource is a mediator for the study of conics and the acquisition of new knowledge that allows the mobilization of mathematical knowledge from perspectives other than graphic approach or common language.
\end{abstract}

Keywords: digital educational resource; semiotic registers; didactic engineering; conical sections 


\section{INTRODUCCIÓN}

Investigaciones en educación matemática revelan frecuentes dificultades que presentan los estudiantes ante determinadas prácticas matemáticas; algunas enmarcadas en las diferentes interpretaciones sobre los sistemas de representación de los objetos matemáticos y su relación con la enseñanza y aprendizaje (Aznar et al., 2016; Duval, 2006). A la Matemática como área primordial de los currículos escolares, junto con Lenguaje y Ciencias se le atribuye una gran responsabilidad en el desarrollo de competencias, conocimientos y habilidades claves para la participación de forma exitosa en los requerimientos que el mundo contemporáneo hace al individuo (Valero, 2017). Esta visión, la hace parte de las políticas de calidad educativa en los países, mostrando preocupación por los desempeños de sus estudiantes en algunas pruebas nacionales e internacionales que realizan estudios comparativos de la calidad de la educación. Una de estas pruebas internacionales, en Matemática, la lidera la Organización para la Cooperación y el Desarrollo Económico (OCDE) conocida como Programa para la Evaluación Internacional de Alumnos (PISA, por sus siglas en inglés) aplicada trianual a estudiantes de 15 años. En general los resultados para América Latina han mostrado desempeños frecuentemente por debajo de la media de todos los países participantes, según los Informes de la OCDE.

Uno de los factores que puede explicar los bajos resultados obtenidos por los estudiantes colombianos en este tipo de pruebas internacionales, es atribuido a la calidad de los profesores, por lo que desde el Ministerio de Educación Nacional en Colombia y algunas Gobernaciones Regionales aúnan esfuerzos por mejorar masivamente la cualificación de sus docentes. Y en ese sentido, diversas investigaciones señalan que se puede avanzar en la educación de jóvenes y en el desarrollo de sus competencias matemáticas en la medida en que sus profesores estén mejor formados (Montes y Gamboa, 2018; Valero, 2017). Sin embargo, también es revelado (Maass et al., 2019), que los avances en la investigación en educación matemática no han tenido el impacto esperado en las prácticas de aula del profesor de matemáticas, en últimas es importante considerar también que el repensar las estrategias de aula que utiliza un profesor influyen en la calidad de los aprendizajes de los estudiantes.

El impacto de la tecnología en la calidad de la educación ha sido investigada por muy diversos autores, sin embargo, según Montes y Gamboa (2018) y Villarreal-Villa et al. (2019), la actualidad colombiana en educación demuestra que, aunque los profesores socialmente aceptan el impacto que ha generado la tecnología, algunos omiten su uso en el campo educativo debido a la falta de capacitación, manejo de estas y porque no reconocen la importancia que podría generar ésta dentro y fuera del aula de clases. Siendo entonces frecuente encontrar espacios académicos donde la matemática sigue un proceso de enseñanza de forma tradicional y lejano de un manejo didáctico que podría llegar a enriquecerse con los recursos que el medio contemporáneo ofrece. Esto sumado, a que los métodos utilizados tradicionalmente en la enseñanza de las matemáticas no han repercutido en resultados positivos y los avances en la didáctica de este saber específico no han logrado modificar de manera decisiva la presentación monótona, complicada y poco interesante para el estudiante y en este sentido cobran gran importancia comprender el uso reflexivo de herramientas tecnológicas y el impacto de los recursos educativos digitales en el desarrollo del profesor de matemática en formación inicial (Trouche et al., 2020).

Investigaciones previas han encontrado que con el uso de la tecnología en la enseñanza y aprendizaje de la geometría en educación superior (García et al.., 2020; Zulnaidi y Syed, 2017) se tienen procesos de enseñanza eficientes (Monroy et al., 2018). y se mejoran los aprendizajes de los estudiantes comparados con aquellos que aprenden sin el uso de recursos tecnológicos, facilitando comprensión y profundización de conceptos geométricos. El estudio de las cónicas como parte de la geometría, se ha abordado usualmente a través de desarrollos teórico-algebraicos mediante metodologías basadas en exposición magistral del profesor y posterior resolución de ejercicios sin embargo, históricamente los resultados de este proceso de enseñanza y aprendizaje no ha sido el esperado e investigaciones desde distintas perspectivas han diseñado diferentes estrategias para su estudio buscando diversificar recursos didácticos con diseños que van desde el uso de material tangible (Villagra y Antunez, 2019) a la incorporación de software de geometría dinámica (Alonso-González et al., 2019; Gómez-Tone, 2019; Peña-Garzón y Rojas, 2019) para algunas construcciones.

Apoyados en Duval (2009), se tiene que "no es posible estudiar los fenómenos relativos al conocimiento sin recurrir a la noción de representación" (p. 25), siendo así se asume de Berger (2010), que hacer y aprender matemática es considerado un comportamiento semiótico. Visto de esta forma, los conceptos fundamentales pertenecientes a la Geometría Analítica, dentro de ellos el estudio de las secciones cónicas, requieren establecer las relaciones necesarias, las formas de representación y las estrategias adecuadas para su enseñanza, lo que se traduce en una necesidad para que dicho concepto sea comprendido, apropiado y asimilado en su totalidad por parte del estudiante. 
Es relevante mencionar que el saber matemático objeto en esta investigación cobra gran importancia puesto que la población objeto de estudio son estudiantes para profesor de matemática, y el saber matemático de las secciones cónicas forman parte de los conocimientos básicos que a través de los lineamientos y estándares básicos presenta el Ministerio de Educación colombiano debe desarrollar el profesor de matemática en ejercicio con los estudiantes de Educación Básica Media, y en los últimos dos grados de escolaridad del joven (identificados como décimo y undécimo grado en Colombia), por lo que se requiere que el estudiante para profesor tenga dominio cognitivo sobre estos.

Este estudio en específico apunta a ofrecer aportes significativos en el estudio de geometría analítica, particularmente en relación con el objetivo de fortalecer el aprendizaje de las secciones cónicas, partiendo de la base que la temática es significativa para la formación de profesores por las aplicaciones dentro de la matemática y fuera de ella, y se fundamenta en la necesidad que el futuro profesor de matemática tenga desarrollos de la temática en los diferentes registros de representación como parte del proceso de aprendizaje completo en la misma, además del apoyo tecnológico como mediador del proceso de enseñar y aprender matemática.

\section{FUNDAMENTOS TEÓRICOS}

Para identificar, analizar y caracterizar los Registros Semióticos en el estudio de las secciones cónicas con profesores en formación inicial, esta investigación se fundamenta teórica y conceptualmente en: la formación del profesorado, la Teoría base de los Registros de Representación Semiótica y el manejo tecnológico a través de recursos digitales de libre uso y con carácter educativo.

\section{Formación del profesorado}

En este apartado se indaga por aportes en la búsqueda de respuestas para el interrogante: ¿Cómo formar profesores de matemática?, investigadores como Godino et al. (2016) y Hill et al. (2008), mencionan que en la formación del profesor se requiere que tenga un conocimiento matemático amplio y sólido, una formación en didáctica sobre la disciplina y un entendimiento de los requerimientos de los estudiantes y del mundo contemporáneo. De esta forma se tiene que una de las claves para mejorar la calidad de la formación del profesor está enmarcada en una estrecha relación entre el conocimiento necesario para enseñar Matemáticas y la práctica del docente; en esta relación juegan un papel importante las tareas y recursos de formación (Borba y Llinares, 2012) que se dirigen a maximizar y optimizar la práctica.

De acuerdo con Ivars et al. (2019) una enseñanza efectiva exige planificar sus objetivos desde el conocimiento de los estudiantes, sus ideas e intereses entre otros; con el propósito de hacer uso de esta información para la toma de decisiones en cuanto al diseño de los ambientes de aprendizaje y en la época actual, un recurso que ha llegado a tornarse como indispensable en la práctica docente son las tecnologías, pues ellas ayudan en el acceso a conocimientos desde otras perspectivas, constituyéndose inclusive en parte del conocimiento en didáctica de la Matemática del profesor; los recursos tecnológicos pueden ser vistos en últimas como soportes didácticos para facilitar la creación de comunidades de aprendizaje.

\section{Plano cognitivo: Registros de Representación Semiótica}

Es marco teórico fundamento en esta investigación la Teoría de los Registros de Representación Semiótica (TRRS) de Duval $(2006,2009,2017)$, y con esta se hace referencia a todos aquellos sistemas de representación que incluyen diferentes sistemas de escritura, como números, símbolos, gráficas, diagramas, esquemas, etc.; y que cumplen funciones de comunicación, objetivación y expresión. Duval (2006) concibe el Objeto Matemático como "el invariante de un conjunto de fenómenos o el invariante de alguna multiplicidad de posibles representaciones" (p. 129), e insta a no confundir el objeto matemático con sus diferentes representaciones.

La relevancia del uso de registros semióticos, como Sistemas Semióticos de Representación, también se apoya en el hecho de que cuando se tiene conciencia de que para referirse a un objeto matemático puede hacerse uso de una gran variedad de representaciones, se comprende entonces que el estudio de las Matemáticas es de naturaleza Semiótica, puesto que los conceptos matemáticos no son accesibles a través de la percepción por los sentidos, sino que es un proceso puramente mental (Godino et al., 2016). Y así entonces, la perspectiva semiótica-cognitiva adoptada por la TRRS, propone afrontar los problemas en el aprendizaje de las matemáticas a partir de los distintos signos (símbolos, iconos, índices, etc.) que se utilizan en esta. Dichos signos, son entendidos como representaciones materiales o externas y no mentales, considerando que la manera necesaria de acceder a los Objetos Matemáticos es a través de sus representaciones. Así, de acuerdo con Duval (2009) hay tres actividades cognitivas inherentes a toda representación: 1) Formación, entendida como construir una marca que sea identificada como una 
representación; 2) Tratamiento, es una transformación interna a un registro utilizando las propias reglas de su sistema; y 3) Conversión, consiste en transformar la representación de un objeto en otra, en un registro diferente.

\section{Recurso Educativo Digital de Acceso Abierto}

La Organización de las Naciones Unidas para la Educación, la Ciencia y la Cultura (UNESCO, por sus siglas en inglés), en el informe emitido en el año 2015, documento Guía Básica de Recursos Educativos Abiertos, define los Recursos Educativos Abiertos como: cualquier recurso educativo (incluso mapas curriculares, materiales de curso, libros de estudio, streaming de videos, aplicaciones multimedia, podcasts y cualquier material que haya sido diseñado para la enseñanza y el aprendizaje) que esté plenamente disponible para ser usado por educadores y estudiantes, sin que haya necesidad de pagar regalías o derechos de licencia. Dentro del contexto educativo colombiano, el Ministerio de Educación Nacional, a través de la oficina de Innovación Educativa con Uso de TIC, y de acuerdo con el Plan Decenal de Educación 2006-2016, e intentando articularse con los planteamientos mencionados por la UNESCO crea la Estrategia Nacional de Recursos Educativos Digitales Abiertos. En la cual define que: Un Recurso Educativo Digital Abierto es todo tipo de material que tiene una intencionalidad y finalidad enmarcada en una acción educativa.

Así, el recurso que haga parte de esta estrategia movilizada por el Ministerio de Educación Nacional colombiano, debe cumplir tres condiciones; 1) ser de carácter educativo: asegurando la relación del recurso con un proceso de enseñanza y aprendizaje, destinado a facilitar la comprensión, la representación de un concepto, una teoría o un fenómeno: 2) ser digital: condición asociada a la propiedad que facilita y potencia los procesos y acciones relacionadas con la producción, almacenamiento, distribución y disposición del recurso en un entorno digital; y 3 ) ser abierto: esta característica responde a los permisos legales que el autor o el titular del Derecho de Autor otorga sobre su obra (Recurso), a través de un sistema de licenciamiento reconocido, para su acceso, uso, modificación o adaptación de forma gratuita.

\section{METODOLOGÍA}

Para este proyecto se utiliza un enfoque mixto con la finalidad de recoger y analizar la información relevante a la investigación de manera descriptiva y estadística (Kelle y Buchholtz, 2015), buscando respuestas para el interrogante de investigación: ¿Cuáles son los diferentes Registros de Representación Semiótica utilizados y que impacten la enseñanza y aprendizaje de las secciones cónicas en profesores en formación inicial?. El diseño es basado en aspectos de la Ingeniería Didáctica (Artigue, 2014) como metodología de investigación cualitativa, complementada con un diseño cuasiexperimental como metodología cuantitativa. Específicamente, se usa un diseño con preprueba-posprueba y grupo experimental. La población objeto de estudio son los estudiantes para profesores de un programa que forma licenciados en matemáticas en 8 semestres en Colombia y la muestra de tipo intencional está constituida por los estudiantes que están matriculados en el curso de Geometría Analítica; este curso en su microcurrículo contempla el estudio de las cónicas. Así los participantes de la investigación son un grupo de 38 estudiantes, ubicados según el plan de estudios en 3er semestre. A este grupo se le aplica la intervención didáctica; el grupo ya ha sido conformado con anterioridad sin la intervención de los investigadores y por motivos diferentes al cuasiexperimento (en este caso, la elección de estudiar la carrera, la asignación de los estudiantes al grupo por parte del Programa o la Universidad).

La metodología se desarrolla en tres fases, la fase 1 con los análisis preliminares epistemológicos y de la enseñanza de las secciones cónicas y de diseño a priori de la estrategia didáctica; en esta fase se aplica al grupo de profesores en formación una prueba inicial $(\mathrm{PI})$ de 12 preguntas, cuyo objetivo es identificar los diferentes Registros de Representación Semiótica utilizados en el estudio que están realizando de las secciones cónicas, y las posibles dificultades que presentan en las actividades cognitivas de: Formación, Tratamiento y Conversión planteadas por Duval. La fase 2 es de experimentación donde se diseña e implementa un recurso didáctico que permita atender las dificultades encontradas a partir de los análisis de la fase 1. Y la fase 3 de análisis a posteriori, validación y confrontación de los resultados obtenidos en las fases 1 y 2 , en esta fase posterior a la intervención didáctica se aplica una prueba final (PF) de 12 preguntas a la muestra con el fin de comparar los resultados obtenidos en ambas pruebas (PI y PF) y determinar el impacto generado por el recurso. Las dos pruebas (PI y PF) se aplican de forma escrita. Estos instrumentos se validan en contenido por juicio de expertos y por pilotaje.

El esquema de metodología utilizado es basado en Artigue (2014) y Kelle y Buchholtz (2015). Dado el enfoque mixto y el cuasiexperimento utilizado como parte del diseño de investigación. Para determinar la normalidad de los datos se aplica la prueba de Shapiro-Wilk para la variable actividad cognitiva presente en la Teoría de los Registros de Representación Semiótica dado que el tamaño de la muestra $n$ de participantes está entre $30<n<50$, teniendo en cuenta un nivel de significancia del $5 \%$ (Walpole, Myers y Myers, 2016). Así mismo, la base de la prueba para la comparación de medias apareadas se realiza con la diferencia de las 
observaciones realizadas a la muestra seleccionada (38 estudiantes). En la figura 1, se presenta de manera esquemática la metodología utilizada. El análisis de los datos cuantitativos se apoyó en el programa SPSS Statistics versión 25 y se usó la regla de decisión dada por un nivel $p$ de significancia con $\alpha=0,05=5 \%$; donde si $p<\alpha$ se acepta la hipótesis del investigador y si $p \geq \alpha$ se acepta la hipótesis nula.

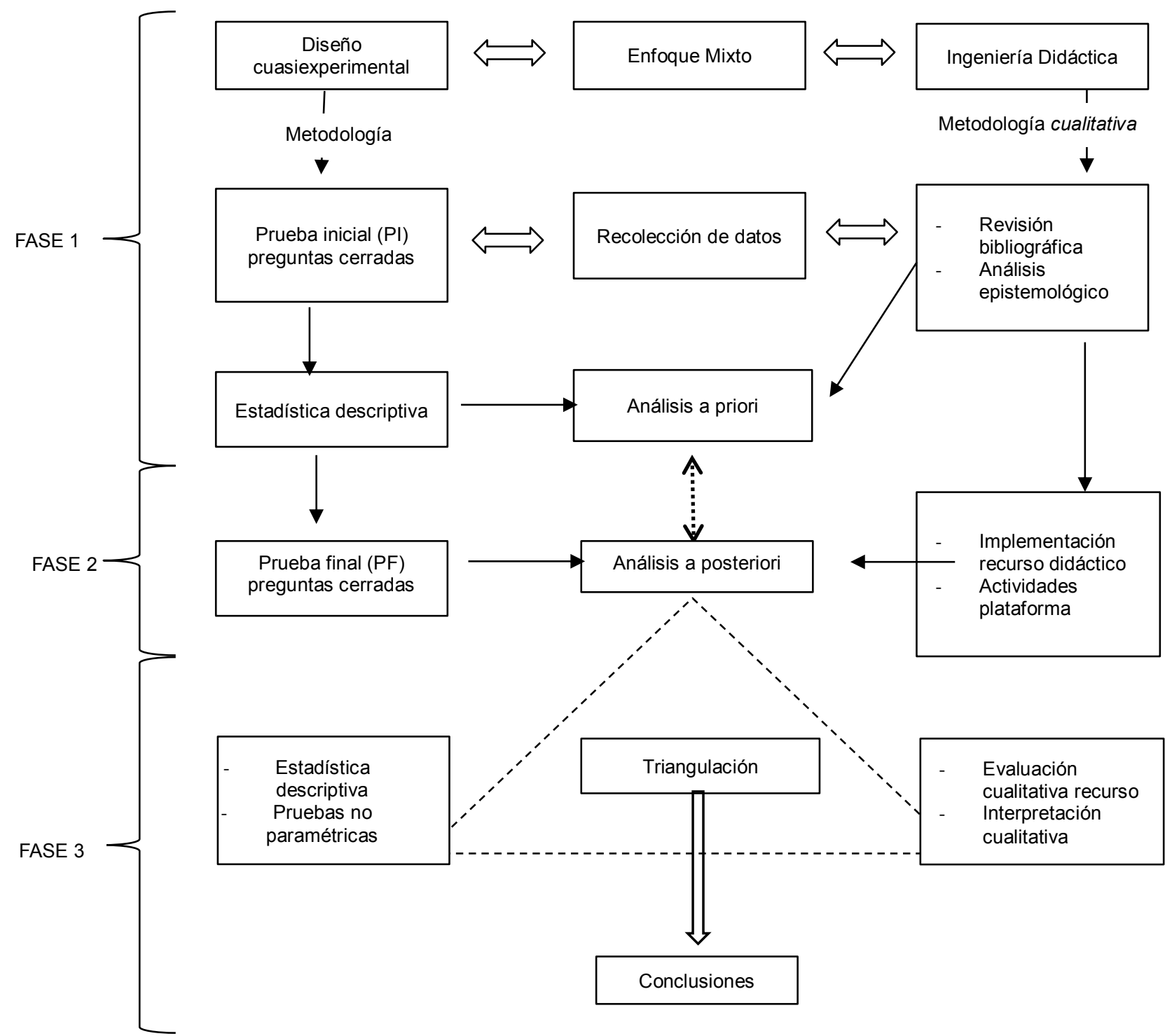

Fig. 1: Esquema de la metodología utilizada.

\section{RESULTADOS}

En este apartado se presenta la caracterización de los registros de representación identificados en el proceso del estudio de las secciones cónicas, y se analiza el impacto sobre los aprendizajes del futuro profesor de matemáticas en el tema de estudio. Los resultados se presentan organizados según los hallazgos en cada una de las tres fases (ver Figura 1) de la investigación.

\section{Resultados obtenidos en la Fase 1}

El análisis en su primera fase se desarrolla, teniendo en cuenta el tratamiento histórico y usual dado al objeto matemático de estudio (cónicas), al saber matemático, los estudiantes y el profesor. Con el arqueo bibliográfico y las observaciones realizadas se evidenció que para el estudio de las cónicas se utilizan tres registros de representación semiótica, que siguiendo a Duval (2006) son identificados como Lenguaje Común, Lenguaje Algebraico y Esquema Gráfico. Se describen a seguir estos registros en el estudio de las secciones cónicas: Lenguaje común: utiliza signos del lenguaje, sintaxis y gramática propia del Castellano, permite realizar explicaciones, y dar definiciones (Duval, 2009). Se evidencia cuando los estudiantes dan la definición de las cónicas como lugar geométrico o como sección cónica, describiendo sus características y elementos; 
Lenguaje algebraico: utiliza signos y reglas propias de la Matemática, y concretamente para este caso del álgebra (Duval 2009, 2017), este se evidencia cuando los estudiantes escriben las ecuaciones canónica y general de una cónica. Y el Esquema gráfico: este registro utiliza el plano cartesiano y la relación entre los ejes coordenados para representar una cónica, permite visualizar las características y elementos propios a este objeto matemático. En la tabla 1 se muestran aplicados a todas las cónicas.

Los resultados de la prueba inicial (PI) evidencian que los estudiantes para la actividad cognitiva de formación solo utilizan una representación semiótica del objeto de estudio, es decir, solo manipulan, coordinan y solucionan actividades enmarcadas en una de las representaciones de la cónicas como su definición como lugar geométrico, como sección cónica, desde su ecuación canónica o general, etc. (ver Tabla 1); para la actividad cognitiva de tratamiento la prueba mostró que los estudiantes presentan dificultades al intentar pasar entre representaciones de los registros semióticos, es decir, cuando se intenta ir por ejemplo, de la representación semiótica de las cónicas como lugar geométrico a la representación como sección cónica, o cuando se intenta pasar de la ecuación general a la ecuación canónica o viceversa; por último, en la actividad de conversión se presentan dificultades en el tránsito entre registros semióticos y en la comprensión e interpretación de las cónicas desde su definición conceptual como lugar geométrico o como formación desde el corte de un plano con un cono. De igual forma, al analizar estos resultados se encuentra que la conversión es la actividad cognitiva que más problemas presenta para los estudiantes.

\section{Resultados obtenidos en la Fase 2}

En la segunda fase, con base en observaciones previas, en el potencial didáctico de la TRRS y en los registros de representación semiótica particulares identificados para cada sección cónica (ver Tabla 1).

Tabla 1: Registros de representación semiótica de las secciones cónicas.

\begin{tabular}{|c|c|c|c|c|c|}
\hline \multirow{2}{*}{$\begin{array}{l}\text { Registro } \\
\text { semiótico }\end{array}$} & \multirow{2}{*}{$\begin{array}{l}\text { Representación } \\
\text { semiótica }\end{array}$} & \multicolumn{4}{|c|}{ Cónica } \\
\hline & & Circunferencia & Elipse & Parábola & Hipérbola \\
\hline \multirow[t]{2}{*}{$\begin{array}{l}\text { Lenguaje } \\
\text { común }\end{array}$} & $\begin{array}{l}\text { Definición como lugar } \\
\text { geométrico. }\end{array}$ & $\begin{array}{l}\text { Conjunto de } \\
\text { puntos en el } \\
\text { plano cuya } \\
\text { distancia a un } \\
\text { punto fijo llamado } \\
\text { centro, es } \\
\text { constante. }\end{array}$ & $\begin{array}{l}\text { Lugar geométrico de los } \\
\text { puntos del plano tales } \\
\text { que la suma de sus } \\
\text { distancias a dos puntos } \\
\text { fijos (focos) es } \\
\text { constante. }\end{array}$ & $\begin{array}{l}\text { Lugar de los puntos } \\
\text { del plano que } \\
\text { equidistan de un } \\
\text { punto fijo llamado } \\
\text { foco y una recta fija } \\
\text { llamada directriz. }\end{array}$ & $\begin{array}{l}\text { Lugar geométrico de los } \\
\text { puntos del plano tal que } \\
\text { la diferencia de sus } \\
\text { distancias a dos puntos } \\
\text { fijos (focos) es } \\
\text { constante. }\end{array}$ \\
\hline & $\begin{array}{l}\text { Definición como } \\
\text { sección cónica. }\end{array}$ & $\begin{array}{l}\text { Sección } \\
\text { perpendicular al } \\
\text { eje de una } \\
\text { superficie cónica. }\end{array}$ & $\begin{array}{l}\text { Curva cerrada con dos } \\
\text { ejes de simetría, que } \\
\text { resulta de cortar un } \\
\text { cono por un plano } \\
\text { oblicuo al eje de } \\
\text { simetría. }\end{array}$ & $\begin{array}{l}\text { Sección que resulta } \\
\text { de cortar un plano con } \\
\text { un cono recto, donde } \\
\text { el plano es paralelo a } \\
\text { la generatriz. }\end{array}$ & $\begin{array}{l}\text { Curva abierta de dos } \\
\text { partes que resulta de } \\
\text { cortar un cono recto por } \\
\text { un plano oblicuo al eje } \\
\text { de simetría. }\end{array}$ \\
\hline \multirow[t]{4}{*}{$\begin{array}{l}\text { Lenguaje } \\
\text { algebraico }\end{array}$} & \multirow[t]{2}{*}{$\begin{array}{l}\text { Ecuación canónica } \\
\text { con centro en }(h, k) .\end{array}$} & \multirow[t]{2}{*}{$\begin{array}{l}(x-h)^{2} \\
+(y-k)^{2} \\
=r^{2}\end{array}$} & $\begin{array}{l}\frac{(x-h)^{2}}{a^{2}}+\frac{(y-k)^{2}}{b^{2}} \\
=1\end{array}$ & $\begin{array}{l}(y-k)^{2} \\
=4 p(x-h)\end{array}$ & $\begin{array}{l}\frac{(x-h)^{2}}{a^{2}}-\frac{(y-k)^{2}}{b^{2}} \\
=1\end{array}$ \\
\hline & & & $\begin{array}{l}\frac{(x-h)^{2}}{b^{2}}+\frac{(y-k)^{2}}{a^{2}} \\
=1\end{array}$ & $\begin{array}{l}(x-h)^{2} \\
=4 p(y-k)\end{array}$ & $\begin{array}{l}\frac{(y-k)^{2}}{a^{2}}-\frac{(x-h)^{2}}{b^{2}} \\
=1\end{array}$ \\
\hline & \multirow{2}{*}{$\begin{array}{c}\text { Ecuación general con } \\
\text { eje focal paralelo al } \\
\text { eje } x \text { y al eje } y \text {. } \\
\qquad \mathrm{g}=0\end{array}$} & \multirow{2}{*}{$\begin{array}{l}x^{2}+y^{2}+D x \\
+E y+F=0\end{array}$} & \multirow{2}{*}{$\begin{array}{l}A x^{2}+C y^{2}+D x \\
+E y+F=0\end{array}$} & $\begin{array}{l}y^{2}+D x+E y+F \\
=0\end{array}$ & $\begin{array}{l}A x^{2}-C y^{2}+D x \\
+E y+F=0\end{array}$ \\
\hline & & & & $\begin{array}{l}x^{2}+D x+E y+F \\
=0\end{array}$ & $\begin{array}{l}C y^{2}-A x^{2}+D x \\
+E y+F=0\end{array}$ \\
\hline \multirow[t]{2}{*}{$\begin{array}{l}\text { Esquema } \\
\text { gráfico }\end{array}$} & $\begin{array}{l}\text { Gráfica como sección } \\
\text { cónica. }\end{array}$ & & & & \\
\hline & $\begin{array}{l}\text { Gráfica en el plano } \\
\text { cartesiano. }\end{array}$ & & & & \\
\hline
\end{tabular}


Se diseña la propuesta de intervención didáctica usando la tecnología, así se materializa un Recurso digital de acceso libre, llamado YilotMath (alojado en una plataforma gratuita: http://yilotmath.herokuapp.com), conformado por tres opciones: Inicio, Contenido y Contacto. Inicio muestra información correspondiente al objetivo de la plataforma, la forma de desarrollo y todo lo referente a su estructura básica y en Contacto se dan formas de comunicación para reusabilidad del recurso entre otros aspectos técnicos.

En Contenido se encuentran los recursos, actividades y propuestas metodológicas diseñadas para el fortalecimiento del aprendizaje del objeto matemático de estudio, esta a su vez se divide en 5 opciones, la primera para los Conocimientos previos en cuanto a la fundamentación teórica previa del saber disciplinar a tratar. En la figura 2 son ilustradas las partes que lo conforman: Contenido, Materiales y Actividades. Las cuatro opciones restantes presentadas en Contenido permiten la interacción con la fundamentación teórica y actividades basadas en la TRRS (Duval, 2006, 2009, 2017), aplicada a las secciones cónicas objeto matemático de estudio, en la figura 3 se muestra para el caso de la Hipérbola las opciones que conforman a su vez las diferentes posibilidades de actividades con las cuales puede ingresar a interactuar el profesor en formación.

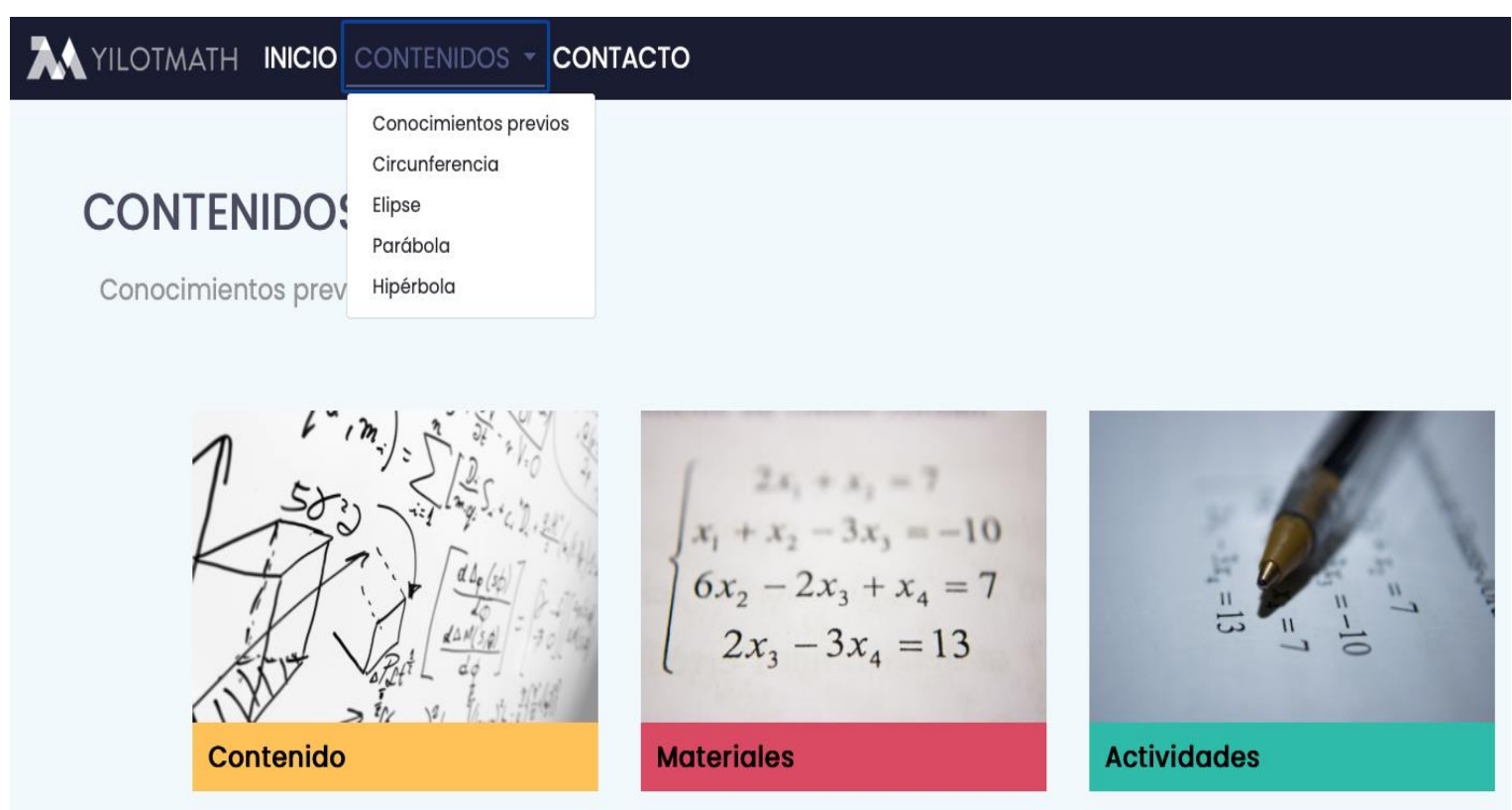

Fig. 2: Actividad del Recurso Educativo Digital de Acceso Abierto: para la opción Conocimientos previos YILOTMATH INICIO CONTENIDOS - CONTACTO

\section{CONTENIDOS}

Hipérbola / Actividades

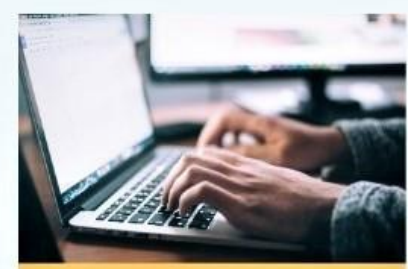

Actividad I hipérbola
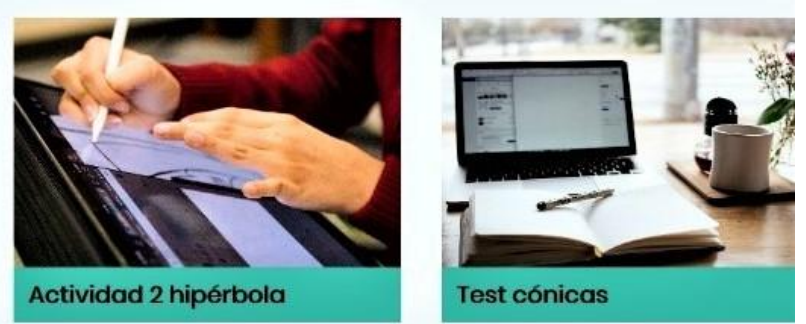

Fig. 3: Actividades del Recurso Educativo Digital de Acceso Abierto: para el Objeto matemático La Hipérbola. 
Todos los elementos del recurso educativo tienen permiso de descarga, dada la concepción de ser un recurso abierto a la comunidad académica. En cada componente del estudio de cónicas se utilizaron los registros semióticos de lenguaje común y algebraico, orientados a través de preguntas que promueven el diálogo y la argumentación del profesor en formación, en el esquema gráfico como registro semiótico se utilizaron dos representaciones semióticas como son el seccionar el cono y las representaciones en el plano bidimensional, en estos últimos casos fue utilizado el programa computacional gratuito para el estudio de la geometría dinámica, el cual tiene gran aceptación en la comunidad de profesores (Zengin, 2018), como lo es GeoGebra.

La selección de cada elemento que conforma el recurso educativo apoya la utilización de diferentes registros semióticos, además se tuvo en cuenta que las herramientas tuvieran la característica de ser de uso libre, contribuyendo al objetivo de que el Recurso educativo a crear sea abierto, esto es, bajo licencia Creative Commons, ejemplo de estas herramientas para el desarrollo del contenido en general son Educaplay, Microsoft Sway, Geogebra, entre otros recursos tecnológicos. El recurso educativo y las actividades dispuestas en él funcionan como apoyo y complemento desde la virtualidad a las clases presenciales, de esta forma, las soluciones que realicen los estudiantes y su interactividad con el recurso están acompañadas y sustentadas en directrices dadas por el docente, en el contexto propio del programa o curso donde se utilice y en el modelo de clase que se tenga (virtual o presencial).

Durante las observaciones, diálogos y revisiones realizadas a las soluciones expuestas por un profesor en formación en una de las actividades de la propuesta (mostrada en la Figura 4) se identifica que, en el desarrollo de la actividad, este hace uso de las dos representaciones semióticas del registro semiótico Esquema gráfico (ver Tabla 1), y en la construcción de su respuesta y análisis utiliza el programa computacional GeoGebra, el proceso que realizó consistía en la manipulación de deslizadores para los parámetros $a, b$ y $c$ de la ecuación de una parábola (mostrada en la Figura 4). Posterior a la manipulación de los parámetros el estudiante profesor debía describir la ecuación canónica de la parábola resultante, con lo que estaba haciendo uso de la representación semiótica Ecuación canónica con centro en $(h, k)$ del registro semiótico Lenguaje algebraico (ver Tabla 1), De manera similar un trabajo exploratorio realizado por Prada et al. (2017), evidenció que los registros semióticos con predominio en estudiantes de dos programas de ingenierías fueron las representaciones algebraicas. Por otro lado, una dificultad encontrada en toda la muestra de estudio estuvo en el caso donde se les pedía en la actividad describir la ecuación canónica de la parábola siempre que la ecuación general tuviera coeficientes con números decimales.

Es importante destacar que algunos de los estudiantes realizaban un proceso de conversión desde la gráfica de la parábola; aquí usaron como mediador semiótico la representación gráfica, encontrando su vértice, foco y directriz para facilitar el encontrar su ecuación canónica. Este proceso de conversión realizado por los estudiantes en una actividad cognitiva de Tratamiento (Duval, 2009).

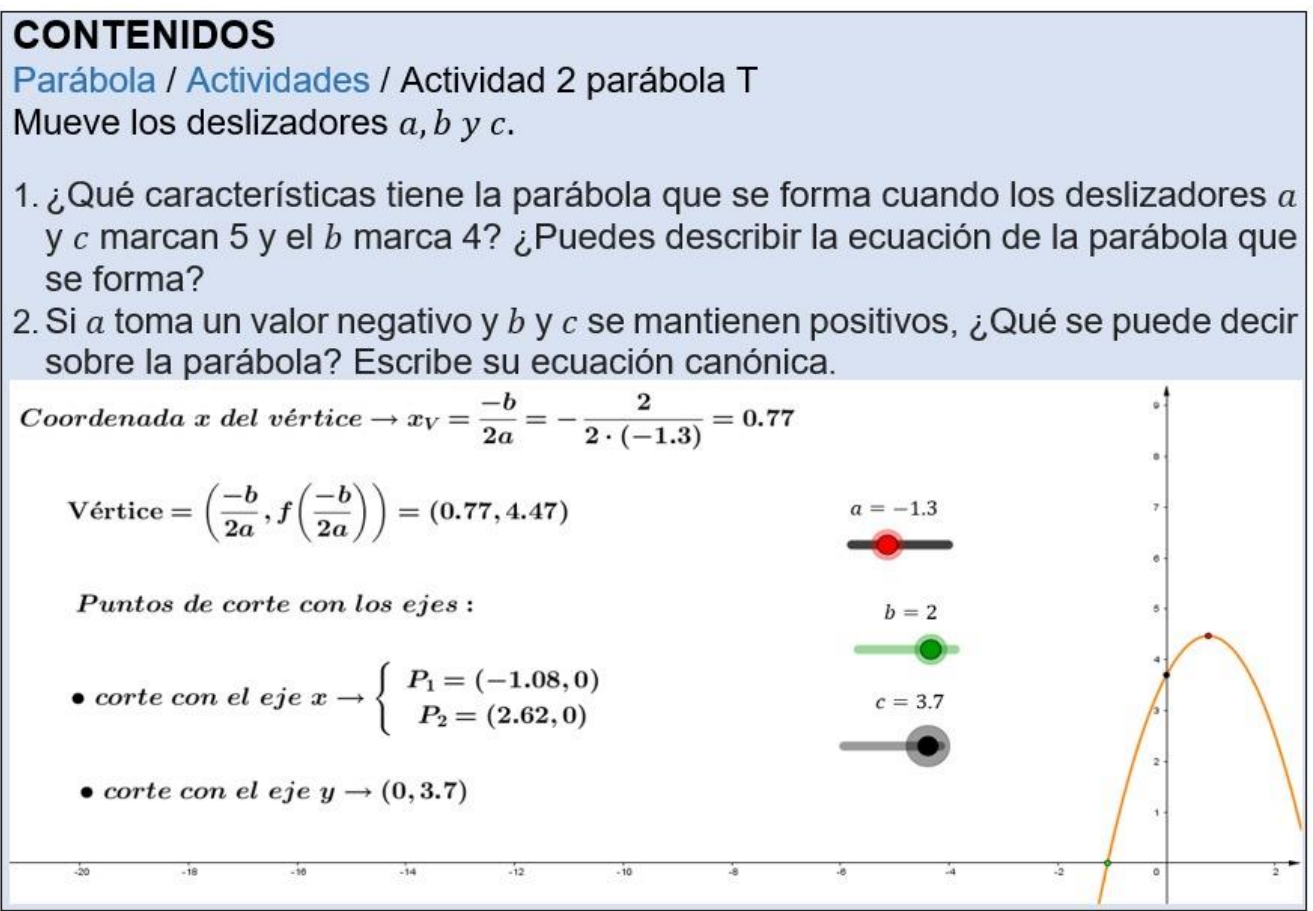

Fig. 4: Actividad de la parábola con representaciones semióticas, (actividad cognitiva Formación) usando GeoGebra en el Recurso Educativo Digital de Acceso Abierto. 
Se evidencia a través de los resultados de la PI y de las observaciones realizadas que desde el punto de vista epistemológico se presentan dificultades en el estudio de las secciones cónicas referidas a la comprensión, representación y tratamiento de las mismas; se asume que esta problemática puede ser resultado de la forma como se enseña el objeto de estudio inmerso en esta investigación, se evidencia que los profesores en formación presentan también dificultades en los conocimientos de los diferentes registros semióticos del objeto matemático estudiado, otros simplemente se centran en la utilización de una o dos representaciones del objeto matemático estudiado y finalmente se hace notar la poca utilización de herramientas tecnológicas como mediadoras para el desarrollo de las actividades cognitivas.

En cuanto al Registro semiótico: Lenguaje común, se muestran y analizan aquí a cuatro (4) individuos pertenecientes a la muestra de estudio, identificados como estudiantes 1 a 4 . Se transcriben algunos apartes de sus respuestas y los hallazgos en lo referente a los registros de representación: definición de una cónica como lugar geométrico y como corte de intersección con el cono. En el caso del Estudiante 1: "Una parábola es una ecuación de segundo grado con una incógnita". De aquí se puede inferir que la definición de la parábola que proporciona está relacionada con objetos matemáticos que ha estudiado o ha aprendido con anterioridad, en este caso, se asocia la parábola con la ecuación cuadrática y se interpreta que asocia la ecuación de este tipo con esta cónica. En la respuesta del Estudiante 2, "La parábola es el conjunto de puntos que están a la misma distancia de un punto fijo y una recta, que forma una curva", se destaca el uso del lenguaje común para definir la parábola a partir de su formación como lugar geométrico, asume las definiciones correctas de eje de simetría y directriz pero no las deja explícitas en su argumentación. Asimismo, al no especificar la forma de la curva queda abierta la posibilidad a una confusión en la interpretación e interiorización del concepto.

Los estudiantes 3 y 4 respondieron: "La circunferencia se forma cuando se pasa un plano de forma horizontal por un cono recto" y "La hipérbola es una cónica que se forma cuando se intercepta un plano en posición vertical con un doble cono circular recto" respectivamente. Aquí se plantean la hipérbola y la circunferencia a partir del corte con un cono. Los estudiantes mencionan de forma apropiada el tipo de inclinación que debe tener el plano que interseca al cono circular recto para la formación de estas. Al hacer las comparaciones de las respuestas dadas por los 4 estudiantes se observa que existe una tendencia a presentar mayores dificultades en la presentación y formulación de una cónica como lugar geométrico, caso contrario para cuando se ve desde el punto vista de un corte con el cono, pues los estudiantes manifiestan definiciones adecuadas y parecen tener claridad de la forma del ángulo e inclinación que debe tener alguna cónica para su formación.

\section{Resultados obtenidos en la Fase 3}

Aquí se presentan los resultados obtenidos en la PI y PF por la muestra objeto de estudio; tras la implementación del recurso tecnológico. Se comparan los resultados desde cada actividad cognitiva y se relacionan categorías de desempeño con un aprendizaje a alcanzar por los estudiantes en las actividades cognitivas de formación, tratamiento y conversión inherentes a toda representación (siguiendo la TRRS), para lo que se diseña una rúbrica, las categorías dadas de Bajo, Medio y Alto, son asociadas a puntuaciones en rangos numéricos mostrados en los paréntesis cuadrados en la Tabla 2.

En los diagramas de caja y bigote, mostrados en la Figura 5, se muestran los resultados de los puntajes obtenidos por los profesores en formación en la PI y la PF en cada una de las Actividades cognitivas enmarcadas en la TRRS y los resultados generales obtenidos en ambas pruebas. Se puede observar que el promedio en la actividad de formación de la PI fue de 1,71 lo que indica que los estudiantes en este componente se encontraban inicialmente en un desempeño bajo (ver Tabla 2), es decir, presentaron dificultades para dar una definición mediante un lenguaje común (registro semiótico) sobre objetos matemáticos; también se observa que existe un punto aislado en el gráfico, lo que representa que un pequeño grupo de estudiantes alcanzó un nivel alto. Por otro lado, en los resultados obtenidos en la PF en la actividad de formación, se observa que se obtuvo un promedio aproximado de 3 indicando que los estudiantes pasaron de un nivel bajo a un nivel medio en este componente tras la implementación de la propuesta de intervención didáctica, es decir, lograron identificar y expresar objetos matemáticos a través de un mismo sistema de representación. Con lo que se evidencia una diferencia positiva en el promedio de la PF y PI.

Para los resultados obtenidos en la PI y la PF en la actividad cognitiva de tratamiento, el promedio obtenido en PI fue de 1,74 puntos, ubicando los estudiantes en un nivel bajo, posiblemente tenían algún aprendizaje sobre las cónicas, pero no lo expresaban con un lenguaje algebraico (registro semiótico) sino a través de un lenguaje común. Se observa también que, en su mayoría los estudiantes se ubican en un nivel bajo - medio. De igual forma, en el diagrama de caja y bigote para PF (ver figura 5), se observa que el promedio general en la prueba fue de 2,84 puntos estableciéndose una diferencia significativa de 1,08 puntos con respecto al promedio de la PI, es decir, los estudiantes lograron un nivel medio en este componente. 
Tabla 2: Rúbrica relación actividades cognitivas y aprendizajes.

\begin{tabular}{|c|c|c|c|c|}
\hline \multirow{2}{*}{$\begin{array}{l}\text { Actividades } \\
\text { cognitivas }\end{array}$} & \multirow{2}{*}{$\begin{array}{l}\text { Aprendizaje } \\
\text { secciones } \\
\text { cónicas }\end{array}$} & \multicolumn{3}{|l|}{ Categorias } \\
\hline & & Bajo $[0,4]$ & Medio $[5,8]$ & Alto $[9,12]$ \\
\hline Formación & $\begin{array}{l}\text { Reconoce, } \\
\text { comprende y } \\
\text { describe el } \\
\text { significado de } \\
\text { las secciones } \\
\text { cónicas en un } \\
\text { mismo sistema } \\
\text { de } \\
\text { representación }\end{array}$ & $\begin{array}{l}\text { El estudiante en este nivel } \\
\text { posiblemente lee } \\
\text { información y define } \\
\text { mediante un lenguaje común } \\
\text { objetos matemáticos como } \\
\text { un lugar geométrico, } \\
\text { relacionándolo con } \\
\text { situaciones cotidianas } \\
\text { dentro de un plano } \\
\text { cartesiano o fuera de este; } \\
\text { sin embargo, puede } \\
\text { presentar dificultades para } \\
\text { describirlo como una } \\
\text { sección cónica. }\end{array}$ & $\begin{array}{l}\text { Describe objetos } \\
\text { matemáticos como un lugar } \\
\text { geométrico. } \\
\text { Identifica en el plano } \\
\text { cartesiano, secciones } \\
\text { cónicas. } \\
\text { Reconoce e interpreta } \\
\text { objetos matemáticos como } \\
\text { secciones cónicas. }\end{array}$ & $\begin{array}{l}\text { Señala información representada en el } \\
\text { plano cartesiano, identificando, } \\
\text { comparando y explicando el significado } \\
\text { de las secciones cónicas. } \\
\text { Utiliza elementos y definiciones, } \\
\text { estableciendo relación particular con } \\
\text { cada cónica. } \\
\text { Relaciona características algebraicas } \\
\text { de las cónicas, sus gráficas y } \\
\text { definiciones en un mismo sistema de } \\
\text { representación. }\end{array}$ \\
\hline Tratamiento & $\begin{array}{l}\text { Representa y } \\
\text { resuelve } \\
\text { problemas de } \\
\text { secciones } \\
\text { cónicas, } \\
\text { mediante la } \\
\text { aplicación de } \\
\text { propiedades, } \\
\text { registros } \\
\text { gráficos y el } \\
\text { uso del } \\
\text { lenguaje } \\
\text { natural y } \\
\text { algebraico } \\
\text { entre } \\
\text { representacion } \\
\text { es semióticas } \\
\text { de un mismo } \\
\text { registro. }\end{array}$ & $\begin{array}{l}\text { En este nivel, el estudiante } \\
\text { probablemente identifica } \\
\text { objetos matemáticos como } \\
\text { lugar geométrico y como } \\
\text { sección cónica, mediante un } \\
\text { lenguaje natural, pero puede } \\
\text { tener dificultades para } \\
\text { expresarlo a través de un } \\
\text { lenguaje algebraico. }\end{array}$ & $\begin{array}{l}\text { Aplica las propiedades de } \\
\text { objetos matemáticos como } \\
\text { lugares geométricos a través } \\
\text { de sus representaciones en } \\
\text { un sistema de referencia. } \\
\text { Utiliza representaciones } \\
\text { gráficas o algebraicas para } \\
\text { resolver problemas prácticos } \\
\text { con secciones cónicas. } \\
\text { Modela ecuaciones } \\
\text { canónicas y generales de } \\
\text { secciones cónicas, usando } \\
\text { un lenguaje algebraico. }\end{array}$ & $\begin{array}{l}\text { Justifica la validez de las propiedades } \\
\text { de las secciones cónicas y las utiliza } \\
\text { en diferentes representaciones } \\
\text { semióticas. } \\
\text { Resuelve problemas mediante el uso } \\
\text { de las propiedades de las secciones } \\
\text { cónicas y usa registros gráficos de } \\
\text { lenguaje natural y lenguaje algebraico. } \\
\text { Describe, representa y compara } \\
\text { objetos matemáticos como secciones } \\
\text { cónicas, infiriendo mediante un } \\
\text { lenguaje algebraico su ecuación } \\
\text { canónica o general. } \\
\text { Identifica secciones cónicas, } \\
\text { transformando su ecuación canónica a } \\
\text { general y viceversa, empleando un } \\
\text { lenguaje algebraico. }\end{array}$ \\
\hline Conversión & $\begin{array}{l}\text { Reconoce, } \\
\text { compara y } \\
\text { explica, objetos } \\
\text { matemáticos en } \\
\text { distintos } \\
\text { registros de } \\
\text { representación, } \\
\text { usando un } \\
\text { lenguaje } \\
\text { natural, } \\
\text { algebraico y } \\
\text { gráfico y realiza } \\
\text { transformacion } \\
\text { es entre estos. }\end{array}$ & $\begin{array}{l}\text { En este nivel, el estudiante } \\
\text { representa lugares } \\
\text { geométricos en el plano } \\
\text { cartesiano, a partir de su } \\
\text { expresión algebraica o } \\
\text { viceversa; sin embargo, } \\
\text { puede presentar dificultades } \\
\text { para interpretar gráficas } \\
\text { como secciones cónicas. }\end{array}$ & $\begin{array}{l}\text { Utiliza expresiones } \\
\text { algebraicas de las cónicas y } \\
\text { propone los rangos de } \\
\text { variación para obtener una } \\
\text { gráfica o una ecuación } \\
\text { requerida. } \\
\text { Reconoce objetos } \\
\text { matemáticos en el plano } \\
\text { cartesiano e infiere su } \\
\text { ecuación canónica o } \\
\text { general. } \\
\text { Compara información gráfica } \\
\text { de secciones cónicas entre } \\
\text { diferentes registros de } \\
\text { representación. } \\
\text { Realiza transformaciones de } \\
\text { objetos matemáticos usando } \\
\text { información de un lenguaje } \\
\text { natural a un lenguaje } \\
\text { algebraico. }\end{array}$ & $\begin{array}{l}\text { Identifica objetos matemáticos como } \\
\text { secciones cónicas y en el plano } \\
\text { cartesiano, empleando un lenguaje } \\
\text { algebraico. } \\
\text { Reconoce y explica las propiedades de } \\
\text { los objetos matemáticos y de sus } \\
\text { transformaciones a partir de diferentes } \\
\text { sistemas de representación. } \\
\text { Selecciona información para resolver } \\
\text { problemas con secciones cónicas, } \\
\text { usando diferentes tipos de } \\
\text { representaciones. } \\
\text { Modela objetos matemáticos, haciendo } \\
\text { uso de un lenguaje natural y algebraico } \\
\text { en diferentes sistemas de } \\
\text { representación. }\end{array}$ \\
\hline
\end{tabular}


Con respecto a la conversión, de la PI se aprecia un promedio general de 2,11 puntos centrándose en un nivel bajo; para la PF se obtuvo una media de 3,08 logrando una diferencia con respecto a la PI de 0,97 puntos. Aunque la diferencia entre las dos pruebas es menor en comparación a las diferencias obtenidas en los otros dos componentes, se observa que en esta actividad cognitiva los estudiantes en general tienen un mejor desempeño. Se logra apreciar, que existe una diferencia significativa en el aprendizaje del objeto matemático tras la aplicación de la PF.

En el último diagrama de caja y bigote de la figura 5 , se comparan los resultados generales de las dos pruebas aplicadas y se observa que en la PI se obtuvo un puntaje promedio de 5,55 lo que ubica a los estudiantes inicialmente en un nivel de desempeño bajo-medio; y en la PF obtuvieron una media aproximada de 9 puntos, situándolos en un nivel alto; evidenciándose progresos importantes.
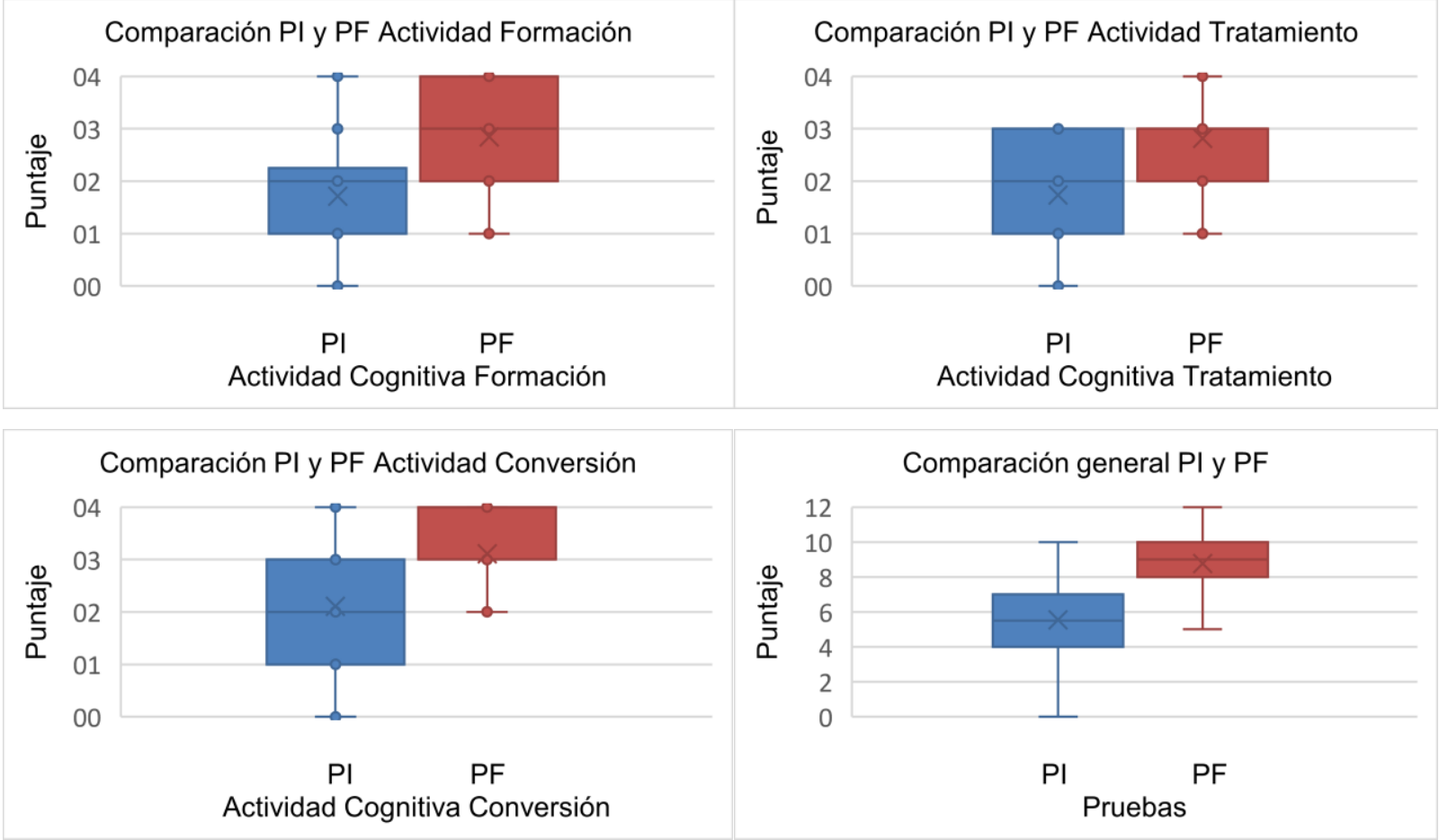

Fig. 5: Diagramas caja y bigotes para los resultados de la Prueba inicial (PI) y Prueba final (PF) obtenidos por los estudiantes para profesores en las actividades cognitivas de formación, tratamiento y conversión.

Por otro lado, el valor $p$ de significancia de la prueba de Shapiro-Wilk corresponde a un $0,005=0,5 \%$, como es menor al establecido para la investigación: se determina que los datos no provienen de una distribución normal. Al no cumplirse los supuestos para la utilización de una prueba paramétrica; para la presente investigación se utiliza la prueba de Rangos y Signos de Wilcoxon. En este caso, como el estadístico de significancia $p$ al estar por debajo de 0,05 y el $z=-5,259$ se toma la decisión de rechazar la hipótesis nula (No existe diferencia significativa en el aprendizaje de los estudiantes - docentes en formación sobre las secciones cónicas utilizando la Propuesta de Intervención didáctica). Por tanto, se asume que sí existe una diferencia significativa entre los resultados obtenidos por los estudiantes profesores en formación en la PI y PF.

\section{DISCUSIÓN}

Los resultados descritos dejan en evidencia las dificultades algebraicas y de conocimientos previos que tienen los participantes. Dicha dificultad puede asumirse a poco trabajo sobre la totalidad de los registros de representación, pues como menciona Duval $(2006,2009,2017)$ en su Teoría, la aprehensión conceptual sobre el objeto matemático sólo se alcanza cuando se estudia sobre varios registros semióticos. Y dado que la muestra objeto de este estudio son profesores en formación, es un asunto importante cómo ellos aprenden y cómo aprenden a enseñar en las condiciones del siglo actual, pues apoyado en el trabajo investigativo de Sánchez-matamoros et al. (2019) la formación del profesorado es cuestión clave pues van a tratar como futuros profesores con estudiantes del siglo XXI, que son cada vez más diversificados y altamente tecnológicos. Teniendo presente que un modelo de enseñanza empírico para un profesor en formación es precisamente como él mismo aprende, con el diseño de la Propuesta de intervención didáctica fundamentada 
en la TRRS de Duval (2009), se encuentra que el entorno de aprendizaje aporta en el inicio de algunas competencias docentes en los estudiantes para profesor, y con esto este trabajo apunta en la misma postura de Roig et al. (2011), en cuanto a que son posibilidades importante de crear oportunidades de aprendizaje para los estudiantes para profesor en el sentido en que le ejemplifican un acto pedagógico para un objeto matemático.

La mayoría de los estudiantes lograron con el uso del recurso educativo realizar transformaciones entre diferentes representaciones semióticas de un mismo registro, interpretar e inferir la ecuación canónica o general de una sección cónica haciendo uso de un lenguaje natural o algebraico, estableciendo las relaciones necesarias y las diferentes formas de representación. Estudios previos de Berger (2010), Duval (2017) y Godino et al. (2016) infieren que esto se logra con estrategias adecuadas en la enseñanza del saber matemático para que pueda ser comprendido, apropiado y asimilado en su totalidad por parte del estudiante.

Entre las dos pruebas aplicadas (PI y PF) existe una diferencia significativa de 3,21 puntos lo que refleja que la implementación y aplicación de la propuesta de intervención didáctica logró generar el impacto esperado y movilizó los aprendizajes de los profesores en formación de manera positiva, lo que es coincidente con escenarios que han encontrado a nivel internacional Instefjord, y Munthe (2017), en cuanto a mejoras significativas en la eficacia de los procesos de enseñanza y aprendizaje cuando en ellos se consideran relaciones con competencias de índole digital, lo que se constituye en un aporte adicional en el apoyo a los procesos de enseñanza y aprendizaje de objetos matemáticos haciendo uso de los diferentes registros de representación semiótica. Este hallazgo, en últimas permite que esta investigación esté en concordancia con los resultados resaltados por Duval $(2006,2009,2017)$ y Godino et al. (2016) en el aspecto en mención.

El resultado de la aplicación de la prueba de Rangos y Signos de Wilcoxon indica que luego de la implementación de la intervención didáctica haciendo uso de la tecnología y del potencial de la TRRS, los resultados para el aprendizaje de las secciones cónicas aumentaron de manera positiva; afirmación que se toma con una confiabilidad del $95 \%$. Esto es, el diseño metodológico permitió evidenciar en los futuros profesores de matemática su producción en relación con los resultados de aprendizaje a partir de la representación semiótica, en este estudio de manera comparable a las conclusiones obtenidas por VillarealVilla et al. (2019), se evidenció de manera cualitativa la alta autopercepción del profesorado en formación objeto de estudio frente a la presencia de competencias digitales en los procesos de enseñanza y aprendizaje, lo que fue favorable en todo el proceso de aprendizaje del objeto matemático.

Dados los resultados de aprendizaje obtenidos haciendo uso de la TRRS y de recursos digitales, estos análisis conducen a consideraciones más generales, en este último sentido de manera similar al estudio realizado por Trouche et al. (2020), sobre la necesidad de equilibrar lo digital y lo no digital, dentro de los procesos de enseñanza y aprendizaje de los estudiantes para profesores, puesto que el uso reflexivo de herramientas tecnológicas permiten el aprovechamiento eficiente de diferentes representaciones promoviendo en el estudiante la construcción y perfeccionamiento de aprendizajes en concordancia con Prada et al. (2017). Un aspecto final para considerar es el que el Recurso diseñado en esta investigación resulta ser la materialización de una estrategia potenciadora para los estudiantes en formación universitaria (profesores en formación inicial en el área de matemáticas) en el desarrollo de habilidades y les permite visualizar, plantear, construir, representar e interpretar de manera más sencilla los problemas espaciales.

Las oportunidades y recursos para el aprendizaje utilizados terminaron siendo motivadores y atractivos para el estudiante para profesor, puesto que está haciendo uso no sólo de recursos digitales, distintos al manejo tradicional del aprendizaje universitario de la Geometría Analítica en este contexto, de solo usar tablero (o pizarra), lo que también le motivaba a ser un diseñador de ambientes enriquecidos de manera digital como alternativas para la enseñanza con sus estudiantes en los momentos de sus prácticas profesionales dentro de la carrera docente, y adicionalmente está siendo consciente que está haciendo uso de una teoría, TRRS, que le será útil como profesor también, lo que aporta en cuanto a lo que Durksen et al. (2017) resaltan como una necesidad el apoyar al maestro en formación y como las motivaciones que este pueda tener en su proceso de formación juegan un papel influyente en la vida como maestro.

La Matemática como un área fundamental en los currículos escolares tiene también la responsabilidad de desarrollar competencias, conocimientos y habilidades claves para la formación y participación del ciudadano de forma exitosa en los requerimientos del mundo contemporáneo. Por lo que el profesor y en particular su formación y desempeño son elementos importantes en esta visión. Luego los aportes que puedan hacerse al proceso de enseñanza y aprendizaje de los profesores en formación constituyen aspectos relevantes en la búsqueda de la calidad educativa.

En este trabajo se analizaron los registros de representación para el estudio de las secciones cónicas, además se identificó el efecto que tiene un recurso educativo digital con un diseño basado en la TRRS de Duval en 
los aprendizajes del futuro profesor de matemáticas. Los resultados obtenidos conducen a las siguientes conclusiones con relación al diseño de la estrategia didáctica: 1) es relevante emplear modelos probados y reconocidos, que contribuyan a desarrollar competencias en el estudiante para profesor en el saber disciplinar, resultado consistente con la evidencia empírica previa, la cual destaca la notable tendencia por la búsqueda de diseños de estrategias que desarrollen y fortalezcan los saberes tanto disciplinares, como pedagógicos y didácticos del futuro profesor, y 2) el uso de recursos tecnológicos para los procesos de enseñanza y aprendizaje con estudiantes para profesores, le destaca el propósito didáctico de la tecnología mostrándola necesaria en el proceso de formación para su futuro ejercicio profesional, pues la utilización de la tecnología en general en las clases de matemáticas sirven como mediadores y transformadores de conocimientos previos y nuevos; y además la evidencia empírica en el plano educativo ha mostrado la tendencia que tiene el profesor en ejercicio profesional a ser un replicador del proceso de formación recibido, por lo que el uso dado a la tecnología con esta población en particular tiene como valor agregado que enriquece el proceso de la práctica ejemplarizante.

En síntesis, este trabajo ofrece un aporte empírico a la formación universitaria del profesor en relación con los principales conceptos estudiados, así como la identificación del uso de modelos alternos al enfoque tradicional de abordar el estudio de las cónicas. Del mismo modo, permite reconocer la importancia del modelo dado por la TRRS para desarrollos de estrategias de enseñanza y aprendizaje y de la tecnología como herramienta mediadora de estos y la necesidad de generar estrategias de aprendizaje que además fomenten ejemplos de enseñanza para el futuro profesor. Lo que fomenta y contribuye en la identificación de patrones de diseño de intervenciones didácticas para el futuro profesor.

\section{CONCLUSIONES}

Los resultados obtenidos con la aplicación de la estrategia didáctica conducen a las siguientes conclusiones: 1) los resultados de aprendizaje obtenidos a través del diseño e implementación de una propuesta de intervención didáctica tecnológica, apoyado en la TRRS de Duval, dan muestra de movilización de saberes matemáticos dentro de intervalos estadísticamente aceptables, y 2) al ser un recurso educativo digital de uso abierto, puede ser reusable, modificable y adaptable a cualquier espacio educativo en cualquier contexto para el aprendizaje y la enseñanza de las secciones cónicas, siendo estas un componente importante de los saberes que aporta la Geometría Analítica al desarrollo de competencias básicas en el área de la matemática escolar.

\section{REFERENCIAS}

Alonso-González, C., Campoy, R., y otros dos autores. Experimentando con GeoGebra y las curvas cónicas en la asignatura Geometría Lineal. Roig-Vila, Rosabel (ed.). Investigación e innovación en la Enseñanza Superior. Nuevos contextos, nuevas ideas. 827-836, Barcelona (2019).

Artigue M., Perspectives on design research: the case of didactical engineering, https://doi.org/10.1007/978-94-017-91816_17. In: Bikner-Ahsbahs A, Knipping C, Presmeg N (eds), Approaches to qualitative research in mathematics education. Springer, New York, 467-496 (2014).

Aznar, A., Baccelli, S., y otros tres autores, Las Funciones Semióticas como instrumento de diagnóstico y abordaje de errores. https://doi.org/10.1590/1980-4415v30n55a18, Bolema, 30(55), 670-690 (2016).

Berger, M., A Semiotic View of Mathematical Activity with a Computer Algebra System. Revista latinoamericana de investigación en matemática educativa, 13(2), 159-186 (2010).

Borba, M.d.C., y Llinares, S., Online mathematics teacher education: overview of an emergent field of research, https://doi.org/10.1007/s11858-012-0457-3, ZDM Mathematics Education,44,697-704 (2012).

Durksen T.L., Klassen, R.M. y Daniels, L.M., Motivation and collaboration: The keys to a developmental framework for teachers' professional learning, https://doi.org/10.1016/j.tate.2017.05.011, Teaching and Teacher Education, 67, 53-66 (2017).

Duval, R., Understanding the Mathematical Way of Thinking - The Registers of Semiotic Representations., Springer International Publishing AG, Switzerland (2017).

Duval, R., Semiósis e Pensamento Humano: Registros semióticos e aprendizagens intelectuais, Editora Livraria da Física, São Paulo, Brasil (2009).

Duval, R., A Cognitive Analysis of Problems of Comprehension in a Learning of Mathematics. https://doi.org/10.1007/s10649-006-0400-z, Educ Stud Math 61, 103-131 (2006).

García, M., J., Eguia, I., y otros dos autores., Implementación y evaluación de actividades interdisciplinares mediante applets dinámicas para el estudio de la geometría. https://dx.doi.org/10.4067/S0718-50062020000100063. Formación Universitaria, 13(1), 63-70. (2020). 
Godino, J., Wilhelmi, M., y otros tres autores. Análisis de la actividad matemática mediante dos herramientas teóricas: Registros de representación semiótica y configuración ontosemiótica, https://doi.org/10.35763/aiem.v0i10.144, AlEM. Avances de Investigación en Educación Matemática, 10, 91-110 (2016).

Gómez-Tone, H., C., Impacto de la Enseñanza de la Geometría Descriptiva usando Archivos 3D-PDF como Entrenamiento de la Habilidad Espacial de Estudiantes de Ingeniería Civil en el Perú. https://dx.doi.org/10.4067/S071850062019000100073, Formación universitaria, 12(1), 73-82 (2019).

Hill, H., Ball, L y Schilling, G., Unpacking Pedagogical Content Knowledge: Conceptualizing and Measuring Teachers' Topic-Specific Knowledge of Students. J. for Research in Mathematics Education, 39(4), 372-400 (2008).

Instefjord, E.J., y Munthe, E., Educating digitally competent teachers: A study of integration of professional digital competence in teacher education, https://doi.org/10.1016/j.tate.2017.05.016, Teaching and Teacher Education, 67, 37-45 (2017).

Ivars, P., Fernández, C., y Llinares, S., A Learning Trajectory as a Scaffold for Pre-service Teachers' Noticing of Students' Mathematical Understanding. https://doi.org/10.1007/s10763-019-09973-4, Int J of Sci and Math Educ 18, 529-548 (2019).

Kelle U., y Buchholtz N., The Combination of Qualitative and Quantitative Research Methods in Mathematics Education: A "Mixed Methods" Study on the Development of the Professional Knowledge of Teachers, , https://doi.org/ 10.1007/97894-017-9181-6_12, In: Bikner-Ahsbahs A., Knipping C., Presmeg N. (eds) Approaches to Qualitative Research in Mathematics Ēducation. Advances in Mathematics Education. Springer, Dordrecht 321 -361 (2015)

Maass, K., Cobb, P., y otros dos autores. Different ways to implement innovative teaching approaches at scale. https://doi.org/10.1007/s10649-019-09920-8, Educ Stud Math 102, 303-318 (2019).

Montes, A., y Gamboa, A., Miradas sobre la calidad de la educación básica en Iberoamérica: Visiones de España y Colombia, https://doi.org/ 10.19053/01227238.8721, Revista Historia de la Educación Latinoamericana, (20)31, 229-244 (2018).

Monroy, A., Hernández, I., A., y Jiménez, M., Aulas Digitales en la Educación Superior: Caso México. https://dx.doi.org/10.4067/S0718-50062018000500093, Formación universitaria, 11(5), 93-104. (2018).

Peña-Garzón, L., y Rojas V., O. Aprendizaje de curvas y superficies de nivel para generalizar familias de secciones cónicas y de superficies cuádricas Roca. Revista Científico - Educacional De La Provincia Granma, 15(3), 1-12 (2019).

Prada N.R., Hernández-Suárez, C. y Jaimes C.L., Representación semiótica de la noción de función: concepciones de los estudiantes que transitan del colegio a la universidad. http://dx.doi.org/10.15765/pnrm.v11i20.1008. R Panorama, 11(20), 33-44 (2017).

Roig, A., Llinares, S., y Penalva, M.C., Estructuras argumentativas de estudiantes para profesores de matemáticas en un entorno en línea. Educación matemática, 23(3), 39-65 (2011).

Sánchez-Matamoros, G., Fernández, C., y Llinares, S., Relationships among prospective secondary mathematics teachers' skills of attending, interpreting and responding to students' understanding., https://doi.org/10.1007/s10649-0189855-y, Educ Stud Math 100, 83-99 (2019).

Trouche, L., Rocha, K., y otros dos autores, Transition to digital resources as a critical process in teachers' trajectories: the case of Anna's documentation work. https://doi.org/10.1007/s11858-020-01164-8, ZDM Mathematics Education, 52, 1243-1257 (2020).

Valero, P., El deseo de acceso y equidad en la educación matemática, https://doi.org/10.17227/01203916.73rce97.126, Revista Colombiana de Educación, (38)73, 99-128 (2017).

Villagra, M., y Antunez A., Enseñanza de curvas cónicas con materiales didácticas. Acta Latinoamericana de Matemática Educativa- Clame, Propuestas para la enseñanza de las matemáticas. Sección 2, 32(2) 312-321 (2019)

Villarreal-Villa, S., García-Guliany, J., y otros dos autores. Competencias Docentes y Transformaciones en la Educación en la Era Digital., https://doi.org/ 10.4067/S0718-50062019000600003, Formación Universitaria, 12(6), 3-14, (2019).

Walpole, R., Myers, S. y Myers, R., Probability \& Statistics for Engineers \& Scientists, 9a Ed, Pearson, (2016).

Zengin Y., Incorporating the dynamic mathematics software GeoGebra into a history of mathematics course, https://doi.org/10.1080/0020739X.2018.1431850, International Journal of Mathematical Education in Science and Technology, 49(7), 1083-1098 (2018).

Zulnaidi, H., y Syed Z., S. N. A., The Effectiveness of the GeoGebra Software: The Intermediary Role of Procedural Knowledge On Students' Conceptual Knowledge and Their Achievement in Mathematics. https://doi.org/10.12973/eurasia.2017.01219a, Eurasia Journal of Mathematics, Science and Technology Education, 13(6), 2155-2180 (2017). 